\title{
Caracterización estructural y electroquímica de materiales útiles como electrodo en baterías recargables de litio
}

\author{
F. GARCíA-ALVARADO , U. AMADOR, M. ÁlVAREZ Y A. MARTíNEZ DE LA CRUZ ${ }^{1}$ \\ Departamento de Química Inorgánica y Materiales. Facultad de CC. Experimentales y Técnicas. \\ Universidad San Pablo CEU. Crta. Boadilla del Monte km 5,300. E-28668 Madrid. \\ ${ }^{1}$ Facultad de Ciencias Químicas. División de Estudios Superiores. \\ Universidad Autónoma de Nuevo León. Apartado Postal 1625. Monterrey. N.L. Mexico.
}

\begin{abstract}
Se ha aplicado la técnica electroquímica que ha dado en llamarse Espectroscopía de Potencial Electroquímico a varios sistemas de intercalación redox para mostrar que ésta se presenta como una herramienta útil para el estudio de las fases presentes en dichos sistemas reduciendo considerablemente el trabajo de síntesis y caracterización estructural de compuestos formados en reacciones de intercalación redox. Puede así determinarse de una forma sencilla el número de fases presentes antes de proceder a su síntesis y posterior caracterización. Por otro lado se presentan algunas de las limitaciones de uso que presenta esta técnica. En particular los margenes de composición determinados no resultan fiables cuando se aplica a compuestos en los que la cinética de reacción es muy lenta o en los que se producen reacciones desplazamiento. De igual forma se ha establecido cuál es el límite de uso de esta técnica $(5.3 \mathrm{~V})$ en el caso de procesos de oxidación que se estudien usando la nueva generación de electrolitos estables por encima de $4.6 \mathrm{~V}$.
\end{abstract}

Palabras clave: Intercalación electroquímica, caracterización electroquímica., espectroscopía de potencial electroquímico

Structural and electrochemical characterisation of electrode materials for lithium rechargeable batteries

The so called Electrochemical Potential Spectroscopy has been used to characterise several intercalation systems. The technique has shown its usefulness to study the different phases that form when an intercalation reaction occurs. Once the existence domains are determined, the work dedicated to the preparation of samples, in order to perform the structural characterisation, is very much shortened. We also present some cases where the above referred electrochemical technique does not yield good results in as much as the existence domains are not well determined. These are the cases of reactions with a very slow kinetic of intercalation and those where displacement reaction occurs. Some problems with experimental set up can also prevent us of using this technique.

Keywords: Electrochemical intercalation, electrochemical characterisation, electrochemical potential spectroscopy.

\section{INTRODUCCIÓN}

Durante la última década el mercado de los pequeños aparatos electrónicos se ha incrementado de forma considerable. Un factor que ha tenido sin duda una gran influencia es que se han comercializado aparatos portátiles que han pasado a formar parte indispensable de las tareas profesionales (por ejemplo teléfonos móviles y ordenadores portátiles). Como en cualquier campo tecnológico el usuario demanda cada vez mayores prestaciones, si bien la sofisticación a la que se ha llegado en estos aparatos no debe ocultar que una de las demandas más importantes a cumplir por un dispositivo móvil es su autonomía. Así, las investigaciones dirigidas a mejorar la capacidad, energía y ciclabilidad de las baterías recargables que les alimentan han experimentado en esta última década un gran auge. En este sentido parece generalmente aceptado que las baterías de litio es una de las opciones más prometedoras. Como ejemplo claro de este futuro puede pensarse en la gradual sustitución de las baterías clásicas Ni-Cd usadas en teléfonos móviles por las que se han desarrollado siguiendo el concepto de baterías ión litio (rocking chair en la bibliografía anglosajona). Los resultados han sido tan espectaculares desde que Sony comercializó la primera de estas baterías en 1991 que ahora las miradas se dirigen hacia el desarro- llo de baterías de este tipo que sean capaces de alimentar un vehículo eléctrico.

A pesar de todo el esfuerzo realizado desde entonces por mejorar los materiales usados por Sony $\left(\mathrm{LiCoO}_{2}\right.$ como electrodo positivo y $\mathrm{C}$, coke o grafito, como negativo) estos parecen ser los más adecuados. La valoración de las prestaciones de un nuevo material pasa inicialmente por una caracterización electroquímica donde se analiza la reacción de inserción de Li como huésped en una estructura anfitrión, M

$$
x \mathrm{Li}+\square \mathrm{M} \rightarrow \mathrm{Li}_{\mathrm{x}} \mathrm{M}
$$

prestando especial atención a la cantidad que se inserta (x), voltaje, cinética y reversibilidad. Estos cuatro aspectos se relacionan directamente con los parámetros que caracterizan a las baterías como son capacidad, energía, potencia y vida media respectivamente. Cuando los resultados no son del todo satisfactorios o, en cualquier caso, se desea su mejora, a menudo hay que recurrir a realizar la caracterización estructural de los compuestos que se forman en el transcurso de la reacción. Como paso previo a esta caracterización debe decidirse qué composiciones merecen ser estudiadas. La propia caracteriza- 
ción electroquímica proporciona la primera pista si se usa lo que Thompson en 1979 (1) nombró como "Espectroscopía de Potencial Electroquímico" (EPE). Con esta técnica puede determinarse el número de fases presentes en el sistema de intercalación y sus márgenes de existencia. Esto sirve como punto de partida para la caracterización estructural de las fases formadas en el transcurso de la reacción. No obstante en algunos sistemas es difícil llegar a resultados satisfactorios debido a problemas característicos de las técnicas electroquímicas y sistemas estudiados.

Pretendemos en el presente trabajo, a través de algunos casos concretos, poner de manifiesto la utilidad de las técnicas electroquímicas para conseguir, en conjunción con técnicas difractométricas, una buena, y eficaz, caracterización de sistemas de intercalación. Se presentan también algunos casos donde la naturaleza del sistema no hace aconsejable la utilización de la EPE. Finalmente pondremos de manifiesto que en condiciones de alto potencial oxidante los dispositivos experimentales pueden interferir en los resultados de la EPE por lo que se hace necesario un control escrupuloso del comportamiento electroquímico de cada uno de los componentes supuestamente "no activos".

\section{EL USO DE LA ESPECTROSCOPÍA DE POTENCIAL ELECTROQUÍMICO}

La medida de la variación de la diferencia de potencial entre los electrodos de una célula con configuración Huésped ( $\mathrm{Li}$, Zn,..)/electrolito/ Anfitrión(M) cuando se le aplica una corriente constante, proporciona en el caso de producirse una reacción de intercalación información sobre la naturaleza de los compuestos cuya composición media es calculada, a través de la Ley de Faraday, y expresada como $\mathrm{Li}_{\mathrm{x}} \mathrm{M}$ por ejemplo si el huésped fuera el ión $\mathrm{Li}^{+}$. Si la reacción produce la formación de una solución sólida, para la cual se supone que los iones litio se encuentran intercalados al azar en un conjunto de posiciones cristalográficas, la variación de E con x es en forma de " $\mathrm{S}$ ", observándose pues una gradual disminución de potencial a medida que aumenta el contenido en litio (2). Por el contrario cuando la reacción de intercalación produce una separación en dos fases, de composición constante, el contenido medio en litio aumenta por variación de las cantidades relativas de ambas fases, pero el potencial permanece constante. En este caso el diagrama E-x traza una meseta $(2,3)$.

No obstante los dos comportamientos ideales descritos son, a veces, difíciles de distinguir en casos reales si bien puede recurrirse a la Espectroscopía de Potencial Electroquímico $(1,4)$. Para ello se somete a la célula a incrementos de potencial de magnitud definida y constante manteniendo dicho potencial durante un tiempo determinado. El sistema genera una intensidad de corriente que es medida en función del tiempo. La intensidad de corriente total en cada paso sirve para calcular el incremento de contenido en litio, por lo que se puede obtener también con esta técnica las correspondiente curvas E-x. Por ejemplo en la figura 1 puede verse un diagrama de este tipo que muestra el comportamiento de una célula de configuración $\mathrm{Zn} / \mathrm{Zn}\left(\mathrm{CF}_{3} \mathrm{SO}_{3}\right)_{2} 1 \mathrm{~mol}$ $\mathrm{dm}^{-3}$ en $\mathrm{PC}+\mathrm{DMSO} / \mathrm{WO}_{3}$ monoclínico, ciclada en condiciones potenciostáticas $( \pm 10 \mathrm{mV} / 6 \mathrm{~h})$ a $25^{\circ} \mathrm{C}$. Se distinguen cinco regiones distintas en base a la diferente pendiente de la curva E-x. Las regiones I ,II y III podrían asignarse a regiones monofásicas mientras que probablemente, aunque más difícil de asegurar, A y B sean regiones bifásicas. La asignación inequívoca puede hacerse si en cada paso de potencial se observa como varía, al

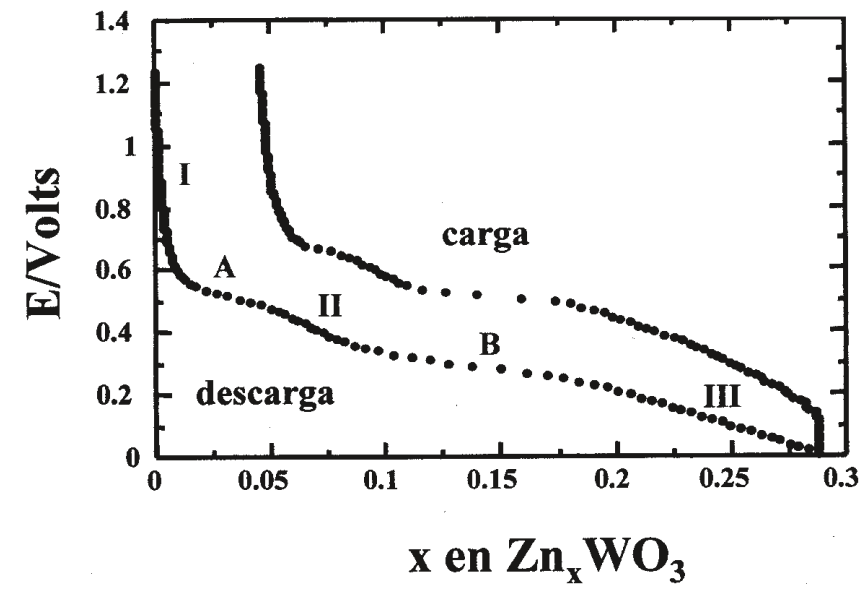

Figura 1.- Variación del potencial de una célula " $\mathrm{Zn} / \mathrm{Zn}\left(\mathrm{CF}_{3} \mathrm{SO}_{3}\right)_{2}$ $1 \mathrm{~mol}$ dm-3 en PC +DMSO/ $\mathrm{WO}_{3}$ monoclínico" en función del grado de intercalación de Zn. Los datos han sido obtenidos a partir de experimentos realizados en condiciones potenciostáticas $( \pm 10 \mathrm{mV} / 6 \mathrm{~h})$ a $25^{\circ} \mathrm{C}$ entre 1.23 y $0 \mathrm{~V}$

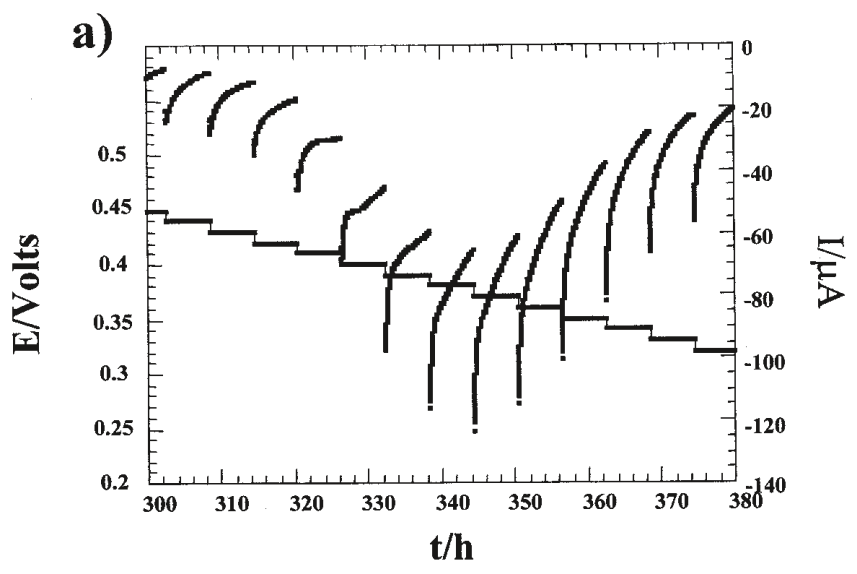

b)

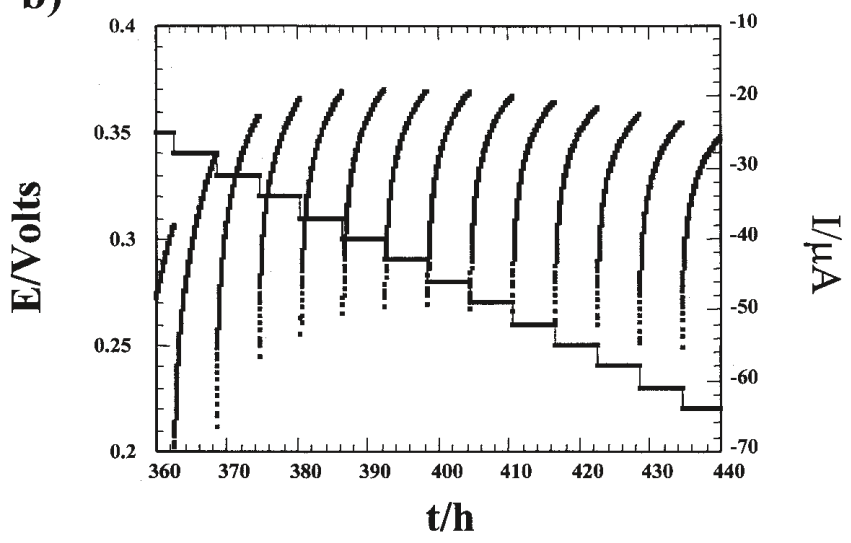

Figura 2.- Detalle del comportamiento de la corriente con el tiempo cuando el sistema $\mathrm{Zn} / / \mathrm{WO}_{3}(\mathrm{~m})$ atraviesa algunas de las regiones mostrada en la figura 1. región bifásica B, b) región monofásica III.

relajarse el sistema, la intensidad de corriente con el tiempo. En la figura 2 puede verse la variación de corriente para tiempos o (o composiciones) próximas a las regiones B y III. En la primera, Figura 2a, se observa que a ambos lados del mínimo de corriente la relajación de la misma sigue comportamientos distintos. Por otro lado en la región III, Figura 2b, las curvas de relajación son idénticas. Estos comportamientos pueden generalizarse a lo 
que se debe esperar para regiones bifásicas y monofásicas respectivamente (4-7). Realizando el mismo tipo de análisis se concluye que también las regiones I y II corresponden a regiones monofásicas mientras que en la región $\mathrm{A}$ encontraríamos la segunda región bifásica del sistema $\mathrm{Zn}-\mathrm{WO}_{3}(\mathrm{~m})(8)$. Preparando ahora tres células con la misma configuración y parando cada una de ellas a composiciones correspondientes a las regiones I, II y III respectivamente conseguimos sintetizar muestras que pueden ser luego caracterizadas por difracción de rayos $\mathrm{X} \mathrm{u}$ otras técnicas difractométricas o espectroscópicas, con objeto de estudiar las estructuras de las únicas tres fases, aunque de contenido variable, existentes en el sistema. El resultado, que ya ha sido descrito previamente por nosotros $(8,9)$ se muestra en la Figura 3. La realización de estos experimentos a diferentes temperaturas constituiría pues una forma relativamente cómoda para determinar el diagrama de fases $\mathrm{Zn}-\mathrm{WO}_{3}(\mathrm{~m})$.

En la figura 4- se muestra el resultado de un estudio similar llevado a cabo en el sistema $\mathrm{Li}-\mathrm{W}_{18} \mathrm{O}_{49}(9)$. En este, aunque se han determinado las distintas regiones existentes aun no se conocen todos los detalles de las estructuras que se forman en las regiones monofásicas ya que nos encontramos ante un ión, $\mathrm{Li}^{+}$, que puede ocupar tres tipos de túneles en los que se pueden alojar más de un litio simultáneamente.

Sirva todo lo anterior, en cualquier caso, como ejemplo de la utilidad que la EPE tiene para caracterizar un sistema cualquiera de intercalación y compárese con el procedimiento alternativo el cuál requeriría la síntesis y caracterización de un alto numero de compuestos con objeto de acotar en primer lugar las zonas bifásicas y monofásicas. Aún así la confluencia de información con la EPE solo sería posible cuando se preparase un numero realmente elevado de muestras.

\section{LAS LIMITACIONES DE LA ESPECTROSCOPÍA DE POTENCIAL ELECTROQUÍMICO}

\section{a) Sistemas con cinética de reacción lenta.}

En el apartado anterior se ha presentado el resultado de aplicar la EPE a dos sistemas distintos. Independientemente de la diferente cantidad de litio que uno u otro inserten y de las diferentes estructuras a las que la reacción de intercalación pueda dar lugar, a efectos de aplicación de la EPE presentan una diferencia muy significativa. Mientras que en la intercalación de $\mathrm{Zn}$ en $\mathrm{WO}_{3}$ el ión que se intercala es divalente, en la de $\mathrm{Li}$ en $\mathrm{W}_{18} \mathrm{O}_{49}$ el ión huésped es monovalente. Este factor diferenciador debe influir claramente en la movilidad del ión huésped en la estructura del anfitrión y por tanto en la cinética de la reacción de intercalación. En la Figura 5 se observan algunos procesos de relajación en el sistema $\mathrm{Li}-\mathrm{W}_{18} \mathrm{O}_{49}$ obtenidos para pasos de potencial de $-10 \mathrm{mV} / 15 \mathrm{~h}$ en una región monofásica. Se observa que el sistema llega en cada paso de potencial, y tras quince horas de relajación, a valores de I próximos a cero. Se alcanza pues el equilibrio en cada paso. Compárese esta situación con la mostrada en la figura $2 b$, obtenida durante la intercalación de $\mathrm{Zn}$ en $\mathrm{WO}_{3}(\mathrm{~m})$. En ésta la relajación en cada paso de potencial se permitió durante 6 horas, si bien cuando se duplica este tiempo el sistema sigue tan alejado del equilibrio como lo está en la situación mostrada en la figura $2 b$.

Si realizamos el análisis de las curvas obtenidas mediante EPE en sistemas que no alcanzan el equilibrio en las condiciones fijadas, aunque el número de regiones mono o bifásicas pudiera ser válido tanto la extensión como los valores absolutos de composición no serían correctos pues en condiciones de

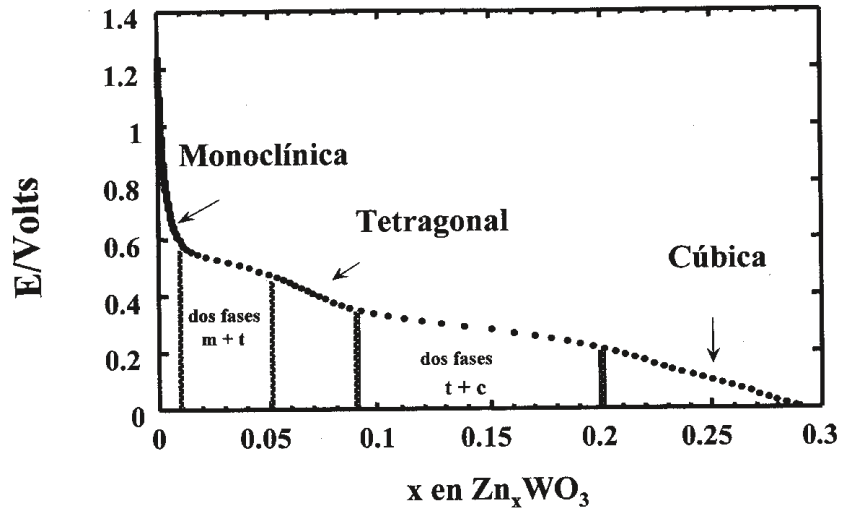

Figura 3.- Diagrama de las fases del sistema $\mathrm{Zn}-\mathrm{WO}_{3}(\mathrm{~m})$ en función del contenido en $\mathrm{Zn}$ a $25^{\circ} \mathrm{C}$. Se han usado tanto técnicas difractométricas como electroquímicas para su elaboración. Se puede observar una relación muy clara entre la forma de la curva E-x y la existencia de regiones bifásicas y monofásicas.

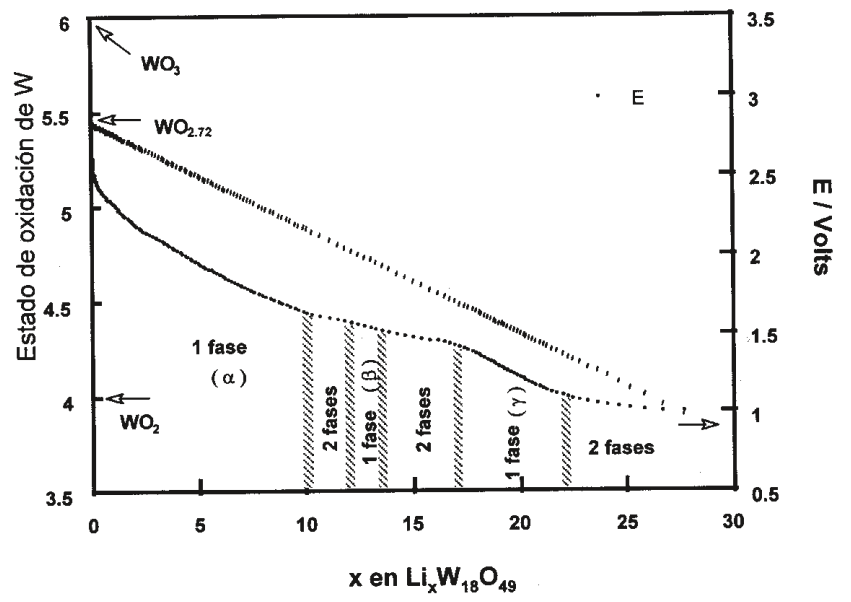

Figura 4.- Diagrama de la fases del sistema $\mathrm{Li}-\mathrm{W}_{18} \mathrm{O}_{49}$ en función del contenido en $\mathrm{Li}$ a $25^{\circ} \mathrm{C}$. Los datos han sido obtenidos por disminución de $\mathrm{E}$ en $10 \mathrm{mV} / 15 \mathrm{~h}$ entre 2.7 y $1 \mathrm{~V}$. Se muestra también cual sería para cada composición el estado de oxidación de W. Se observa que la inserción de litio hasta $1 \mathrm{~V}$ produciría la reducción de todo el $\mathrm{W}$ a $\mathrm{W}^{4+}$.

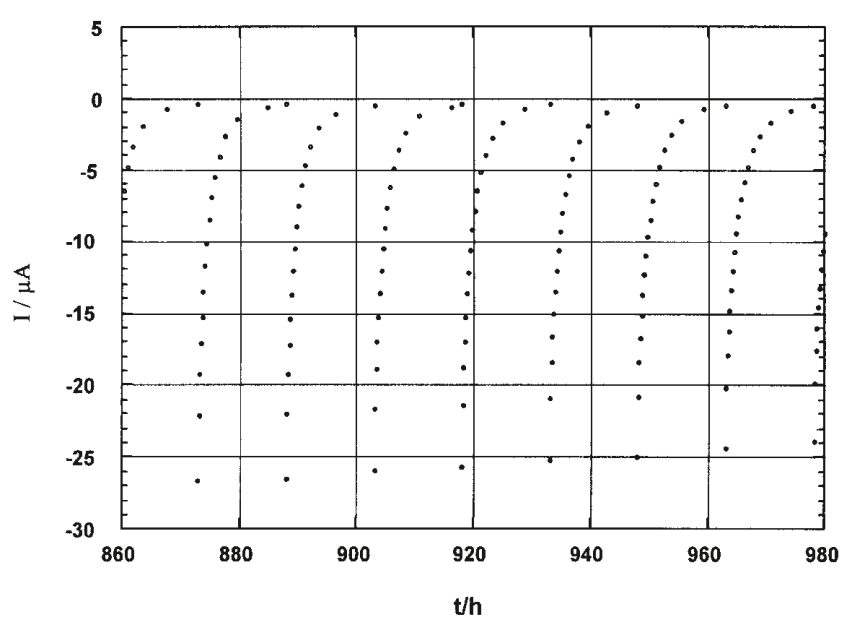

Figura 5.- Detalle del comportamiento de la corriente con el tiempo cuando el sistema $\mathrm{Li} / / \mathrm{W}_{18} \mathrm{O}_{49}$ de la figura 4 atraviesa una region región monofásica. $\mathrm{Al}$ final de cada paso de potencial (tras 15 horas) se observa que la corriente cae a un valor muy próximo a cero indicando que el sistema, cuyo potencial se va disminuyendo en pasos de $10 \mathrm{mV}$, está alcanzando de nuevo el equilibrio en cada paso. 
equilibrio siempre se observaría un mayor grado de intercalación. Aunque por lo general siempre es posible encontrar las condiciones que aseguren el equilibrio dichas condiciones podrían ser poco prácticas. Baste pensar que se ha estimado que para los valores típicos de $\mathrm{D}_{\mathrm{Li}}$ encontrados en óxidos y calcogenuros, desde $10^{-8}$ a $10^{-12} \mathrm{~cm}^{2} \mathrm{~s}^{-1}$, y tamaños de partícula del orden de $20 \mu \mathrm{m}$, el tiempo necesario para que el centro de un grano- de geometría esférica- alcance el $90 \%$ de la concentración correspondiente al paso de potencial impuesto, varía desde minutos hasta días respectivamente (4). En este último caso y dependiendo del intervalo de potencial barrido, un experimento que proporcionara valores absolutos sobre limites de existencia duraría más de un año!. El problema podría aliviarse si se controla el tamaño de partícula pues una disminución hasta los $2 \mu \mathrm{m}$ disminuiría la duración del experimento a unos cuantos días. En cualquier caso para iones divalentes en estructuras como las anteriormente citadas el coeficiente D podría bajar hasta los $10^{-19} \mathrm{~cm}^{2} \mathrm{~s}^{-1}$ haciendo cualquier intento por aplicar la EPE inútil.

\section{b) Presencia de reacciones de desplazamiento.}

El sistema $\mathrm{Cu}_{\mathrm{x}} \mathrm{V}_{2} \mathrm{O}_{5}(\mathrm{x}=0.85-1)$ nos permite presentar otro ejemplo donde la aplicación de la EPE no produce los resultados que serían deseables. En la Figura 6 se muestra el comportamiento, descarga y carga, de una célula con configuración Li/ $1 \mathrm{~mol} \mathrm{dm}^{-3} \mathrm{LiPF}_{6}$ en $\mathrm{EC}+\mathrm{DMC}(3: 1) / \mathrm{Cu}_{0.85} \mathrm{~V}_{2} \mathrm{O}_{5}$ obtenido en condiciones potenciostáticas $( \pm 10 \mathrm{mV} / 6 \mathrm{~h})$ en el intervalo de voltaje 1-3.6 V. Entre otras cosas se observa que en carga aparentemente se consigue extraer más litio del que se introduce. La incongruencia surge porque se está adjudicando toda la carga transferida a la extracción de litio cuando en realidad por encima de $3 \mathrm{~V}$, aproximadamente, se produce la extracción de $\mathrm{Cu}^{+}$. Al abrir la célula se detecta de hecho que la superficie del litio se ha cubierto de un polvo rojizo que se correspondería con la electrodeposición de $\mathrm{Cu}^{+}$como $\mathrm{Cu}$. El fenómeno, ya observado con anterioridad (10) no tendría implicación alguna si no fuera porque también se produce aunque no se proceda a la carga de la célula. Si la aparición de cobre se produce tambien durante la intercalación de litio se puede pensar en una reacción de desplazamiento $\mathrm{Li} \leftrightarrow \mathrm{Cu}$. Resulta evidente que, al no poder controlarse apropiadamente este proceso, la composición de las fases obtenidas por "intercalación" queda indeterminada cuando se intenta utilizar la EPE para estudiar este sistema. Aunque el caso de este bronce de cobre y vanadio pueda parecer anecdótico las reacciones de desplazamiento son comunes habiendose encontrado en otros sistemas como por ejemplo en $\mathrm{Li}-\mathrm{Ag}_{2} \mathrm{~V}_{4} \mathrm{O}_{11}$. En este último caso el desplazamiento es tan acusado que se observa un cambio importante entre la forma de la primera descarga y la de la primera carga (11).

\section{c) Oxidaciones hasta alto potencial}

Durante muchos años la mayoría de los materiales de intercalación, y así también los propuestos como electrodos en baterías recargables de litio, han sido estudiados a potenciales comprendidos entre 0 y $4.5 \mathrm{~V}$ respecto a $\mathrm{Li}^{+} / \mathrm{Li}$. Tras el descubrimiento de un nuevo electrolito estable por encima de $5 \mathrm{~V}$, (12), algunos materiales en los que no había sido posible extraer todo el litio presente fueron investigados de nuevo. Se consiguió así extraer electroquímicamente todo el litio de $\mathrm{LiMn}_{2} \mathrm{O}_{4}$ para obtener $\lambda-\mathrm{MnO}_{2}(13)$, y por primera vez se obtuvo por extracción de $\mathrm{Li}$ en $\mathrm{LiCoO}_{2}$ un óxido binario de $\mathrm{Co}^{4+}$, $\mathrm{CoO}_{2}(14)$. Las nuevas expectativas crean también nuevos pro-

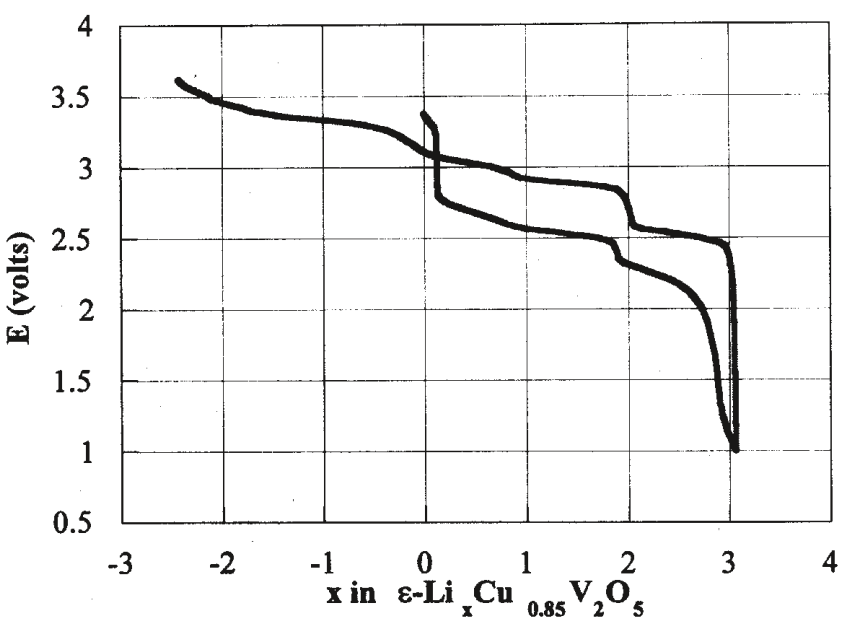

Figura 6.- Curva E-x del sistema $\mathrm{Li}-\mathrm{Cu}_{0.85} \mathrm{~V}_{2} \mathrm{O}_{5}$ obtenidas de un experimento potenciostático $( \pm 10 \mathrm{mV} / 5 \mathrm{~h})$. El efecto observado en carga, sobre $3.2 \mathrm{~V}$, indica que a potenciales altos el cobre está siendo extraído.

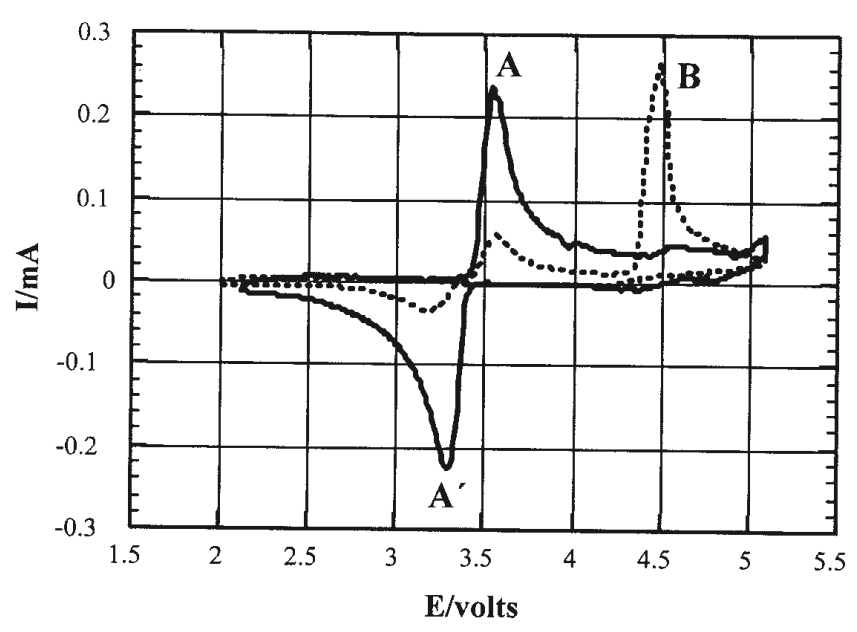

Figura 7.- Respuesta del sistema $\mathrm{LiFePO}_{4} /$ / Li a un barrido de potencial de $\pm 10 \mathrm{mV} / 0.5 \mathrm{~h}$. La curva de trazo discontínuo se obtuvo de una célula que utilizó como electrodo una mezcla de $\mathrm{LiFePO}_{4}$ carbón conductor y EPDTP como aglomerante. La de trazo contínuo se diferencia de la primera en que el aglomerante utilizado fue Kynarflex®.

blemas. Nosotros hemos detectado que usando la nueva familia de electrolitos constituídos por la sal $\mathrm{LiPF}_{6}$ disuelta en carbonato de etileno y dimetil carbonato, algunos elementos de nuestro dispositivo experimental producen una señal de oxidación. En la figura 7 pueden verse las señales correspondientes a la oxidación y reducción ( $\mathrm{A}$ y $\mathrm{A}^{\prime}$ ) en $\mathrm{Li}^{-}-\mathrm{FePO}_{4}$ tal y como describe Padhi et al (15). Los procesos son reversibles aun cuando a alto potencial se observa una señal de oxidación (B), que no tiene su homólogo en reducción, pero que desaparece cuando, en lugar de etilen-propilen-dien-terpolímero como aglomerante se usa Kynarflex®.

En cuanto a la estabilidad de estos nuevos electrolitos en la figura 8 puede verse que cuando se intenta oxidar $\mathrm{LiNiPO}_{4}$ nos encontramos con un proceso irreversible a unos 5.8. aunque pudiera pensarse que este proceso corresponde la oxidación irreversible del $\mathrm{LiNiPO}_{4}$ en realidad corresponde a la oxidación irreversible del electrolito, $\mathrm{u}$ otro componente de la célula, puesto que la señal es la misma si cambiamos el mate- 
rial activo por $\mathrm{LiMnPO}_{4}$ o si incluso el experimento se realiza sin material activo. Así pues, con células de configuración similar a las usadas por nosotros (material activo + carbón conductor+ Kynarflex/ $\mathrm{LiPF}_{6}$ en $\left.\mathrm{EC}+\mathrm{DMC} / \mathrm{Li}\right)$, el uso de la técnica de EPE se encuentra limitado a alrededor de $5.5 \mathrm{~V}$ valor para el que la corriente oxidativa ya es considerable. No obstante para la mayoría de los sólidos que pueden actuar como anfitrión este valor de potencial máximo de trabajo resulta más que suficiente para llevar a cabo una correcta caracterización electroquímica que apoye la caracterización estructural de compuestos de intercalación.

\section{CONCLUSIONES}

Hemos mostrado que la técnica electroquímica que ha dado en llamarse Espectroscopía de Potencial Electroquímico se presenta como una herramienta útil para el estudio de las fases presentes en sistemas de intercalación reduciendo considerablemente el trabajo de síntesis y caracterización estructural de compuestos formados en reacciones de intercalación de iones en compuestos anfitrión. No obstante se han puesto también de manifiesto las limitaciones que esta técnica tiene cuando se aplica a compuestos en los que la cinética de reacción es lenta o en los que se producen reacciones desplazamiento. De igual forma se ha establecido cuál es el límite de uso de esta técnica en el caso de procesos de oxidación que se estudien usando la nueva generación de electrolitos estables por encima de $4.6 \mathrm{~V}$ $\left(\mathrm{LiPF}_{6}\right.$ en $\left.\mathrm{EC}+\mathrm{DMC}\right)$.

\section{AGRADECIMIENTOS}

Los autores agradecen la financiación recibida de la CICYT a través del proyecto MAT98-1053-C04-04 y de la Universidad San Pablo CEU (proyectos 8/97 y 5/98)

Agradecen también los comentarios y sugerencias del Dr. Alario-Franco a buena parte del trabajo aquí presentado.

\section{BIBLIOGRAFÍA}

1. A. H. Thompson “ Electrochemical potential spectroscopy: a new electrochemical measuremet", J. Electrochem. Soc. 126, 608 (1979).

2. W.R. McKinnon, "Insertion electrodes I: Atomic and electronic structure of the hosts and theirinsertion compounds" en "Solid State Electrochemistry". Ed. por P.G. Bruce, Cambridge University Press. Cambridge 1995, cap. 7, pag. 187

3. W. Weppner y R.A. Huggins, "Determination of the kinetic parameters of mixed-conducting electrodes and applications to the system Li3Sb", J. Electrochem. Soc., 124, 1569 (1977).

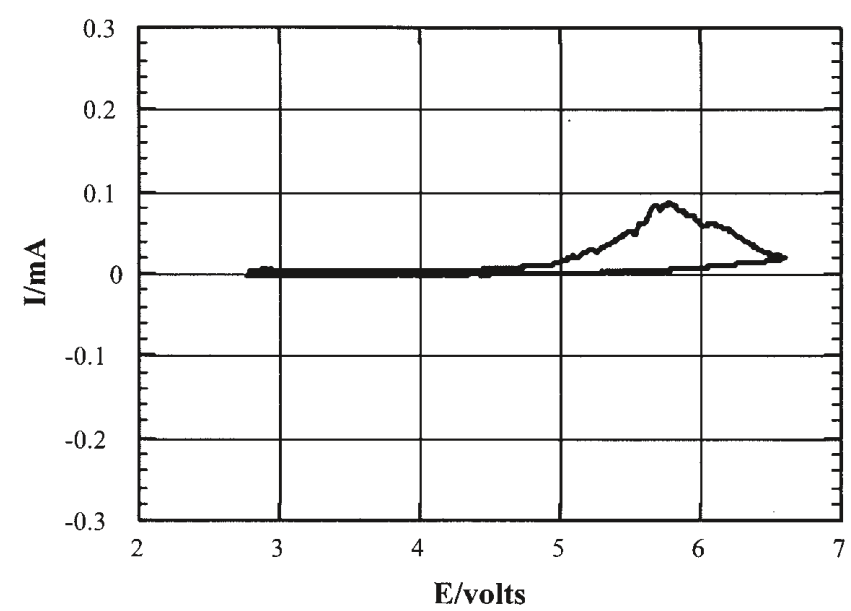

Figura 8.- Respuesta del sistema $\mathrm{LiNiPO}_{4} /$ / Li a un barrido de potencial de $\pm 10 \mathrm{mV} / 0.5 \mathrm{~h}$. No se observa ningun proceso reversible. El máximo a $5.8 \mathrm{~V}$ corresponde la oxidación del electrolito.

4. Y. Chabre, "Electrochemical spectroscopy and in-situ diffraction studies of intercalation compounds" NATO ASI Series 305, 181,(1993).

5. Crank "The mathematics of diffusion", Oxford University Press, Oxford 1967

6. C. J. Wen, B.A. Boukamp, R.A. Huggings, W. Weppner, “ Thermodynamic and mass transport properties of LiAl”, J. Electrochem. Soc. , 126, 2258 (1979).

7. J. Philibert, "Atoms movements; Diffussion and mass transport in solids", Les Editions de Physique, Les Ulis, (1991).

8. A. Martínez de la Cruz, L.M. Torres-Martínez, F. García-Alvarado, E. Morán, y M.A. Alario-Franco, " Formation of new tungsten bronzes: electrochemical zinc insertion in $\mathrm{WO}_{3}{ }^{\prime \prime}$, J. Mat. Chem. 8, pags. 1805-1807 (1998).

9. A. Martínez de la Cruz, " Intercalación electroquímica de algunos iones monovalentes y divalentes en $\mathrm{W}_{18} \mathrm{O}_{49}$ y $\mathrm{WO}_{3}$ “. Tesis Doctoral,Universidad Complutense, Madrid 1997.

10. F. García-Alvarado, J.M. Tarascon y B. Wilkens, " Synthesis and electrochemical study of new copper vanadium bronzes and of two new $\mathrm{V}_{2} \mathrm{O}_{5}$ polymorphs: $\beta^{\prime}$ - and , $\epsilon '-\mathrm{V}_{2} \mathrm{O}_{5}^{\prime \prime}$, J. Electrochem. Soc., 139, 3206-3214 (1992).

11. F. García Alvarado y J.M. Tarascon, "Lithium intercalation in $\mathrm{Ag}_{2} \mathrm{~V}_{4} \mathrm{O}_{11}$ ", Solid State Ionics 73, 247-254 (1994).

12. D. Guyomard y J.M. Tarascon, "A new electrolyte composition for the $\mathrm{Li}_{1+\mathrm{x}} \mathrm{Mn}_{2} \mathrm{O}_{4} /$ carbon rechargeable cells", Proc. of the Electrochemical Society Meeting, Toronto 1992. Ed. por The Electrochemical Society Inc., Pennington (1992).

13. D. Guyomard, J.M. Tarascon,"Rechargeable $\mathrm{Li}_{1+\mathrm{x}} \mathrm{Mn}_{2} \mathrm{O}_{4} /$ carbon cells with a new electrolyte composition. Potentiostatic studies and applications to practical cells" J. Electrochem. Soc.140, 3071 (1993).

14. G.G Amattucci, J.M. Tarascon, L.C. Klein, " $\mathrm{CoO}_{2}$, the end member of the $\mathrm{Li}_{\mathrm{x}} \mathrm{CoO}_{2}$ solid solution" J. Electrochem. Soc. 140, 1114-1120 (1996).

15. A.K. Padhi, K.S. Nanjudaswamy, S. Okada, C. Masquelier y J.B. Goodenough," Effect of structure on the redox couples in iro phosphate", $190^{\text {th }}$ Elctrochemical Soc. Meeting, San Antonio Texas, Octubre 1996. Ed. por The Electrochemical Society Pennington (1998). 